\title{
Routing Protocols for AANET
}

\author{
Gurumekala T, Indira Gandhi S, Senthil Sivakumar M
}

\begin{abstract}
Ad hoc network is an infrastructure less, selfconfiguring and dynamic network where the nodes are able to forward the information to other nodes based on connectivity and routing algorithm they follow. Recently, the concept of this ad hoc networking has been introduced among aircrafts for providing in-flight communication and to manage increased flow of data produced by civil aviation. The aircraft communication can be established either by satellites or cellular based systems. Utilizing satellites for the communication is very expensive and prone to high propagation delay. The cellular based systems provide direct link to aircraft with minimum cost and less delay. As the line-of-sight range of cellular systems is limited, the aircraft over the oceanic regions is unable to communicate with ground stations. Aiming at overcoming the demerits associated with aforementioned technologies for aircraft communication, the Aeronautical Ad hoc networks has been developed, which creates a ad hoc network among aircrafts where each aircraft is self-aware nodes and communicates with ground stations and other aircrafts irrespective of their flight region. AANET shares has some similarities with existing wireless ad hoc networks whilst having unique challenges in supporting greater mobility, size of network, node density and bandwidth limitations. Because of this unique challenges, routing in this AANET is a difficult task. In this paper, various routing algorithms for AANET with its merits and demerits has been thoroughly studied. Finally, the unsolved problems and research issues of routing in AANET are identified.
\end{abstract}

Index Terms- Aeronautical Ad hoc Network, in-flight communication, satellite based systems, cellular based systems.

\section{INTRODUCTION}

The AANET is an emerging research area, which enables the communication between air-air and air-ground beyond the sender's radio range. Each aircraft is self-ware and acts as relays, receivers and senders for data transmission [1]. In the continental areas, the in-flight communication is feasible with both cellular system and satellite systems. The cellular based systems provide direct link to aircraft with minimum cost and less delay. But, the line-of-sight range of cellular systems is limited, the aircraft flying over the oceanic regions or remote Polar Regions is unable to communicate with ground stations. For connecting with ground while flying over such regions, aircraft can make use of satellite-based systems [2]-[4], which provide a link between two transceivers by using satellites as relay. Satellite-based systems can cover wider area at relatively high speed, but at the expense of high cost and long delay. The Fig. 1 shows the typical architecture of an aeronautical environment where the communication is taken place among three layers.

In Mobile ad hoc networks (MANET), the mobile nodes collaborate with each other to form dynamic network without any centralized infrastructure. With this autonomous self-organizing characteristics, the mobile nods can freely move anywhere.

Revised Manuscript Received on December 15, 2019.

Gurumekala T, Madras institute of Technology, Chennai, India.

Indira Gandhi S, Madras institute of Technology, Chennai, India.

Senthil Sivakumar M, Vignan's Foundation for Science, Technology and Research, Guntur, India.
VANET is a subset of MANET, where the mobile nodes are moving vehicles approximately 100 to 300 meters of each other to stay in connection and form a network with a wide range. As vehicles move out of the range of signal and fall out of the network, other can join in, connecting vehicles to one another. AANET is a variation of VANET, where mobile vehicles are self-aware aircrafts which construct communication between air-air and air-ground infrastructure.

Although, AANET shares some common characteristics with other ad hoc networks, it gets differentiated because of several critical design considerations such as greater mobility, mobility model, node density, time-varying channels, poor link reliability and quick topology changes. Node movements in MANET and VANET are relatively slow when comparing with AANET. According to [5], an aircraft is capable of moving at a speed of $30-460 \mathrm{~km} / \mathrm{h}$, and this impact severely in several challenging communication design problems [1]. In the case of mobility model, MANET and VANET motilities are predictable to some extent. In AANET, though aircrafts fly in predetermined path, for safety and security purposes the path may be recalculated. Choosing the mobility model accordingly is a noticeable issue. Moreover, the aircrafts are scattered over the sky with several kilometers distance between aircrafts and because of the high speed of aircrafts and link outages the topology changes quickly.

The rest of the paper is organized as follows. Section II deals with routing challenges in AANET. Section III covers various routing algorithms for AANET. Section IV provides insight on open research challenges and Section $\mathrm{V}$ concludes this paper.

\section{ROUTING CHALLENGES IN AANET}

The AANET's unique features demand the researcher to construct new routing algorithms as the MANET or VANET routing algorithms can't be adopted into different scenarios of AANET. The following factors make the routing process difficult.

1. High mobility: The mobility degree of aircrafts is very high ranging from $350-300 \mathrm{~m} / \mathrm{s}$. This higher degree of mobility leads to unpredictable mobility pattern.

2. Frequent topology changes: Due to the greater mobility, the topology is rapidly changing than MANET and VANET. Other factors for frequent topology changes are aircraft platform failure and link outages. Whenever the aircraft platform fails, the link with connected aircraft fails.

3. Sparse distribution of the ground stations: The number of ground infrastructure is minimum and also the line-ofsight is limited. Therefore gaining in-flight communication from the ground station is a difficult task [5],[6]. 


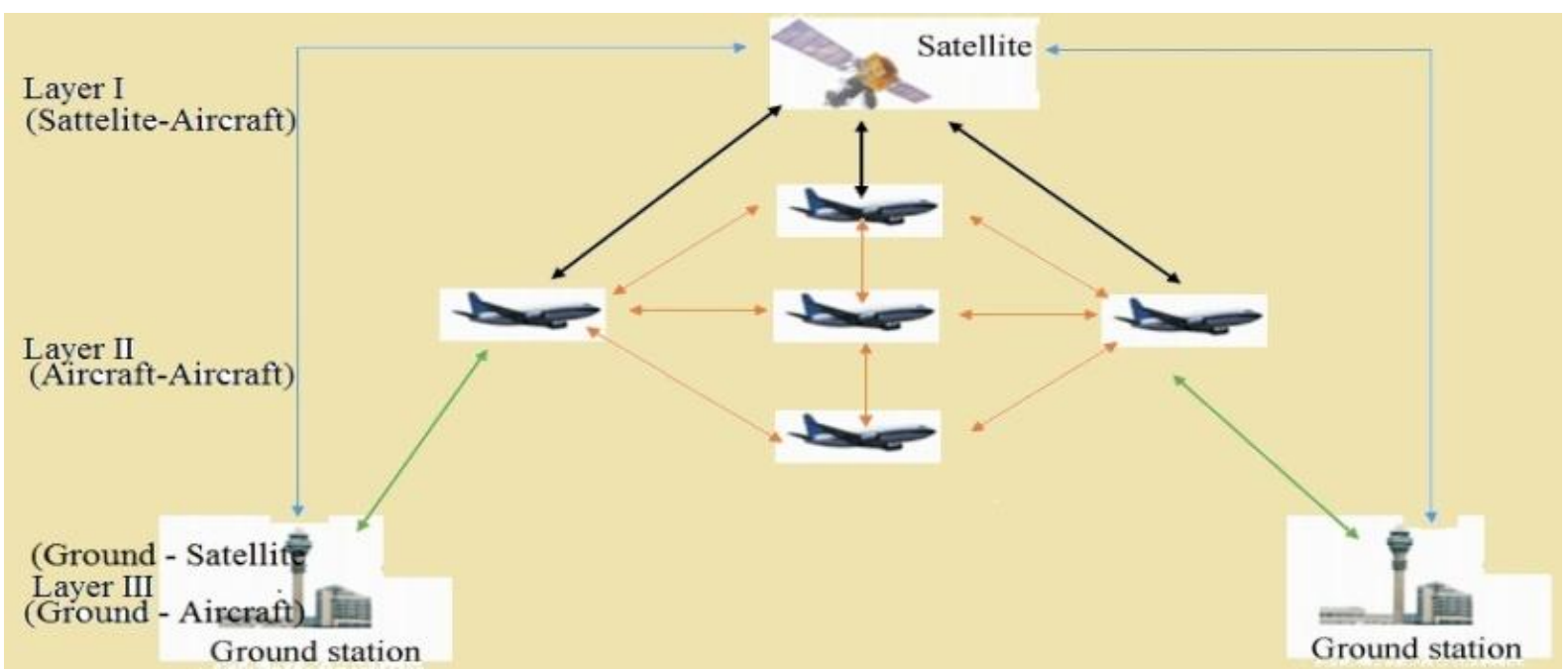

Fig.1. typical architecture of an aeronautical environment

4. Limited bandwidth: AANET has character of long communication distance and limited bandwidth of in area of $\mathrm{U} / \mathrm{VHF}$ band. This factor demands the effective utilization of bandwidth resources [7].

5. Node Density: The aircrafts are scattered in sky. The distance between aircrafts is several kilometers. So the transmission range of each aircrafts needs to high to stay in contact with other aircrafts.

\section{Routing Protocols in AANET}

\section{GLSR: Geographic Load Share Routing}

The goal of this protocol is to come up with multiple path between source and destination to mitigate congestion the in moderate and high density TDMA based aeronautical ad hoc networks. GLSR extends Greedy Perimeter Stateless Routing protocol for wireless networks. In GPSR, the packets are forwarded to geographically closest neighbor of destination [15] - [17]. Each packet arrives at a sender node will be stored in the transmission queue. If the packet arrival rate at sender node is higher than the number of slots assigned to the link between available between source and detonation, then the queuing delay of packets will be increased, eventually results in drooping of packets. This problem is overcome in GPSR by maximizing the packet's advance towards destination and minimizing the queuing delay. The packet's advance is the difference between distances of source and neighbor towards destination. The simultaneous achievement of minimized queuing delay and maximized packets' advance is not possible.

The GLSR protocol considers speed of advance metric of packet to find the appropriate neighbor towards destination. The speed of advance metric denotes the ratio between packet's advance and queuing delay. A sender node before transmitting the packet, first estimates the speed of advance of neighbors on the way to destination. The node with maximum speed of advance is chosen as the next hop for forwarding packets to destination. In this way, GLSR achieves load sharing among all neighbors.

\section{AeroRP: Aeronautical Routing Protocol}

AeroRP [19] is a domain specific geolocationassisted routing protocol designed for dynamic airborne ad hoc networks. The main focus of AeroRP is to find out efficient and stable path for air-air and air-ground communication to route data packets amongst aircraft nodes (ANs) and back to a ground station (GS). The selection of stable path is achieved by incorporating specialized relay nodes (RNs), which equipped with enhanced communication capabilities to assist the routing of data by the aircrafts. Each aircraft may use other aircrafts or RNs as next hops in order to forward the packets to their destination in case of assigned aircraft may not be within transmission range of the ground station or target aircraft within a stipulated time.

The basic operation of AeroRP consists of two phases. In the first phase, the airborne nodes acquires knowledge of its neighbors and makes a list of available neighbors at any given point in time. Two different mechanisms are employed to aid the neighbor discovery, which includes broadcasting beacon messages periodically and storing geo-path information in actual data packets. The second phase of this protocol deals with neighbor discovery, which attempts to find the appropriate next hop to forward the data packets to destination. Each aircraft maintains neighbor table and by periodically updating this neighbor table with the use of method in first phase, each node knows the location of destination. The proper next hop is chosen based on the smallest Time to Intercept (TTI) value to forward each data packets. TTI is computed as follows:

TTI $=[([$ euclidean distance between neighbor and destionation)-Transmission Range])/(Relative Velocity of the neighbor)]

Smallest time to intercept (TTI) indicates the time for a node to be within transmission range of the destination if it continues on its current trajectory. The TTI is the primary metric which is used for selecting the next hop for packet transfer to destination. The sender first calculates the TTI value of its neighbor by using the neighbor table to know whether it is in the transmission range of the destination or not and select the next hop.

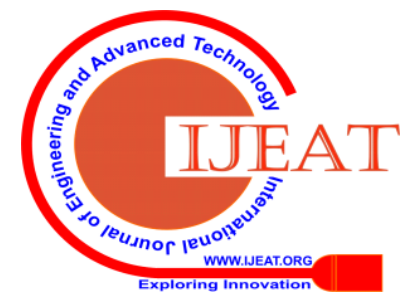


There is a special case that the nodes which are in the transmission range of destination don't need to travel in the same direction of destination. To incorporate this situation the TTI is again refined based on the moving direction of the neighbor node, TTI is categorized as zero TTI and Negative TTI. The Zero TTI indicates that the neighbor node is not in the transmission range of the destination and moving away from the destination. The negative TTI indicates that the neighbor node is in the transmission range of the destination and moving towards the destination.

AeroRP works in three mode while forwarding the packets. They are Ferry, Buffer and Drop. In Ferry mode, the local node (node having holding the packet) keeps the packet in queue indefinitely until it discovers the neighbor node with lowest TTI than the local node. In Buffer mode, the local node keeps the packet with definite time until it finds the neighbor node with lowest TTI than the local node. In Drop mode, the packets in the local node are dropped.

\section{PLAR: Path Link Availability Routing}

The PLAR routing proposes new topology control mechanism to increase the routing path duration by using the aircraft densities along with the topology construction methods in highly mobile aeronautical ad hoc networks [24]. The philosophy behind this protocol is, increasing the path duration results in lower probability of link break up when aircrafts is fast moving. In order to attain this scope, it combines the proposed topology control mechanism with OLSR (Optimized Link State Routing) [25]. This routing introduces two mobility models for aircrafts in AANET considering the flight direction. Three topology control mechanisms are designed to come up with the stable, unstable and controlling the topology based on the stability of the link respectively. The assumptions made by this protocol are as follows,

1. The link is established between two is only when he distance between two nodes should be less than or equal to the transmission range of that two nodes.

2. The link between two high speed nodes remains active when the velocity of nodes are equal.

3. The link between two high speed nodes breaks up when the velocity of nodes changes according to time.

Based on the above assumptions, PLAR frames two mobility models.

Mobility Model 1: The link established by two aircrafts in same direction ensures the higher probability of keeping the path long time than in opposite direction.

This mobility model minimizes the probability of link break up only for multi-hop routing. In the case of smaller density aircraft in the same direction won't ensure the long path duration. This neglected situation is covered in the second mobility model.

Mobility Model 2: The newest link created between two aircrafts in the opposite direction increases the probability of keeping the link for longer time than the old link existed on that air lane.

Based on the framed node mobility model, three topology control mechanisms has been designed based on the link stability.

Mechanism 1: Long Time Available Link Recognition

This method searches for the long time available links between two nodes move in same direction. For that, the stability of link is assessed and compared with the threshold link stability. The threshold link stability is calculated as follows:

Threshold link stability $=(2 *$ transmission range of nodes $) /([$ velocity of nodes- $(3 *$ normal distribution of velocity of nodes)*time period])

After the link creation between two nodes in same direction, the number of beacon messages transferred between that nodes is noted down and if that value is greater than the calculated threshold stability value, then that link is identified as long time available link.

Mechanism 2: Short Time Available Link Recognition

This method identifies the link established between two nodes in same or opposite direction as shot time available links when the number of transferred beacon messages of less than the threshold stability. Whenever greater mobility leads the AANET, large number of unstable link persists.

Mechanism 3: Topology control based on link stability Whenever there is a change in network topology that change must be communicated to all other nodes $i$ an effective way. In order to accomplish this task, stable node that is, node with long time available link is selected to broadcast the topology changes. To avoid excessive flooding because of too many stable, an agent selection algorithm is employed here. This agent selection algorithm chooses the highly stable to disseminate the topology change information.

After selecting the stable links, stable nodes and effective way for topology information dissemination, the routing is carried out similar to OLSR. The OLSR uses MPR (Multipoint Relaying) to avoid broadcasting duplicate messages in network [25]. The MPR set is a set of aircrafts which is created in AANET based the density of aircrafts and link stability. The nodes in MPR set is the minimum set of one-hop nodes which covers two-hop nodes. Thus routing through MPR set ensures the network coverage.

\section{HSRP: Hierarchical Space Routing Protocol}

HSRP aims at providing efficient and reliable communication in AANET with the use of node movement features. This protocol has been designed based on the zone routing protocol (ZRP) for the mobile ad hoc networks [25] that works based on the zones of the node. ZRP is a hybrid routing which combines both proactive (IARP) and ondemand routing protocols (IERP), where IARP is a kind of proactive link-state protocol that keeps track of the routing information for nodes which are available inside the zone. The IERP is a reactive routing protocol which maintains the route discovery and route maintenance services depending upon the connectivity examined by the IARP. IERP is enabled when the destination of the node is outside of the stipulated zone. For routing beyond the zone, BRP (border broadcasting resolution protocol) is employed that makes use of routing information provided by the IARP and follows query control mechanisms to identify the neighbor zone [26]-[31].

By inheriting aforementioned features of ZRP, HSRP has been framed, in that the zones of aircrafts is decided based on the aircraft flow rate and maximum spatial radius which in turn directs to fall in either proactive (IARP) or reactive (IERP) protocols. 
This protocol takes into account the aircraft flow rate, speed of aircraft, aircraft density, and flight free stream velocity and aircraft dangerous density as the node movement features. The aircraft flow rate is described as the number of passing aircrafts in a certain point at unit interval, flight speed denotes the velocity of aircrafts, aircraft density is the number of aircrafts on the unit length of air path at particular time, the flight free stream velocity refers to the speed of air traffic flow when there is no aircraft, the aircraft dangerous density indicates number of aircraft on a air path at particular time with minimum distance.

The routing process is carried out in three phases. The first phase is aircraft flow rate determination, where the values of all node movement features is determined and based on the value of flight free stream velocity and aircraft dangerous density, the relationship between aircraft flow rate, speed of the aircraft and aircraft density is assessed. The core idea behind the HSRP protocol lies in dynamically changing the update frequency of beacon messages according to aircraft flow rate, in which beacon message interval is calculated as a non-periodic function of aircraft flow rate. Whenever greater mobility exist in AANET, aircraft density and aircraft flow rate is larger, thus more neighbor state needs to be updated, improving the update frequency of beacon messages.

The second phase is for dynamic correction of spatial radius. The reason for dynamically correcting the maximum spatial radius is to reduce the routing control overhead of both proactive and on-demand routing protocol. Routing among the same radius for a long time irrespective of aircraft flow rate affects either IARP or IERP. So, the maximum spatial radius is either decreased or increased according to the change of aircraft flow rate. Thus, equally balancing the routing overhead of IARP and IERP, the performance of overall routing is maximized in AANET.

The third phase involves in choosing the suitable routing method based on the dynamically calculated value of the maximum spatial radius. If the destination aircraft of the packet is inside the stipulated spatial radius then, the IARP is initiated by looking into the routing table which has necessary routing information of all nodes within this radius. If the destined aircraft is outside the maximum spatial radius, the IERP is initiated, which takes care of route discovery and route maintenance with the help of information provided by IARP and forward the packets to the border of the zone. After that, BRP plays role in sending the packets to destined aircraft with the use of query control mechanisms.

Another optimization technique is employed in HSRP protocol for avoiding no uniformity of spatial radiuses of aircraft node. This non-uniform character of aircraft node results in redundant routing information, thus creates a chance for routing overhead. In order to avoid the problem of spatial coverage, space generation algorithm is designed, where the node with maximum spatial node is selected as the management node based on the aircraft flow rate and node state information. The selected management node broadcasts link state messages to invite other nodes joining in the newly created space. The management node is changed when three condition meets. One is, there is a change in the spatial radius of the management node, second is the management nodes are in communication range of other nodes and final one is, a node is not in the any space and able to create its own space. This process avoids the problem associated with spatial coverage.

\section{RESULTS DESCRIPTION}

The AANET is delay sensitive and highly dynamic multi-hop network environment. The unique nature of this environment poses critical challenges towards achieving fine-tuned applications. The following are unresolved problems in airborne network.

Gateway Discovery: The gateway for in-flight communication in ground infrastructure is sparsely distributed. The gateway acts a bridge between ground and aircrafts ad hoc networks. If an aircraft's suffers to discover the gateway, the air-air communication will get affected more. In such situation, acquiring the connectivity from right gateway is still being open problem in AANET.

Node Density: The aircrafts are scattered over the sky with very long distant. Because of the greater mobility nature of aircrafts, the density of aircraft over the sky will not be uniform. The number of aircrafts to self-configure the dynamic network may be continuously changing. Routing in such unstable environment should ensure the connectivity irrespective of node density. So matured routing among moderate and low density aircraft is still being an open problem.

Bandwidth Utilization: The variation of aircrafts location tends to have difference in channel quality even though, all aircrafts in same frequency and same transmission rate. So, the effective channel assignment and utilization and link quality estimation is an open problem in AANET.

Frequent Topology Changes: Because of the greater mobility of aircrafts, the topology is often gets changed in AANET. The right prediction about mobility pattern will assist to cope up with the topology changes. But the intelligent mobility prediction with $3 \mathrm{D}$ is still an unsolved problem in AANET.

\section{CONCLUSION}

The emerging AANET area plays vital role in air traffic safety, civil aviation and route planning over sky. Because of its critical design challenges such as mobility, number of nodes, size of the network, routing in AANET is becoming toughest problem to solve. In this paper, various widely used routing method has been thoroughly studied. AeroRP, GRAA, A-GR, DMDR and MQSPR have considered mobility aspects of AANET. NoTe-TBR is the only protocol which has considered the node density aspect of AANET. GRAA, A-GR and MQSPR have taken into account the topology changes of AANET. GLSR, A-GR, DMDR and MQPSR have considered the bandwidth limitation. The PLAR estimates the duration of link availability for selecting routing path. The HSRP protocol traces the movement of aircraft nodes and select the path accordingly. The routing method which considers all design issues is still in research.

\section{REFERENCES}

1. Vivek Kumar, Arti Rana, Sanjay Kumar Aircraft Ad-hoc Network (AANET), International Journal of Advanced Research in Computer and Communication Engineering Vol. 3, Issue 5, May 2014. 
2. A. Jahn et al., "Evolution of aeronautical communications for personal and multimedia services," IEEE Commun. Mag., vol. 41, no. 7, pp. 36-43, Jul. 2003.

3. O. Ercetin, M. O. Ball, and L. Tassiulas, "Modeling study for evaluation of aeronautical broadband data requirements over satellite networks," IEEE Trans. Aerosp. Electron. Syst., vol. 41, no. 1, pp. 361-370, Jan. 2005.

4. E. Lutz et al., "Development and future applications of satellite communications," in Emerging Location Aware Broadband Wireless Ad Hoc Networks. New York, NY, USA: Springer, 2005, pp. 231246

5. Hengyang Zhang, Xiaoping Chen, Bo Zheng, Yequn Wang " Analysis of Connectivity Requirement for Aeronautical Ad hoc Networks", 2011 International Conference on Electronic \& Mechanical Engineering and Information Technology.

6. Hua Li, Bo Yang, Cailian Chen and Xinping Guan, "Connectivity of Aeronautical Ad hoc Networks",IEEE Globecom 2010 Wireless Networking for Unmanned Aerial Vehicles.

7. E. Sakhaee, A. Jamalipour, and N. Kato, "Aeronautical ad hoc networks," in Proc. IEEE Wireless Commun. Netw. Conf. (WCNC), Las Vegas, NV, USA, 2006, pp. 246-251.

8. L. Lei, D. Wang, L. Zhou, X. Chen, and S. Cai, "Link availability estimation based reliable routing for aeronautical ad hoc networks," Ad Hoc Netw., vol. 20, pp. 53-63, Sep. 2014

9. E. Sakhaee, A. Jamalipour, and N. Kato, "Multipath Doppler routing with QoS support in pseudo-linear highly mobile ad hoc networks," in Proc. IEEE Int. Conf. Commun., vol. 8. Istanbul, Turkey, 2006, pp. 3566-3571.

10. D. Johnson and D. Maltz, "Dynamic source routing in ad hoc wireless networks," in T. Imielinski and H Korth, editors, Mobile Computing, Chapter 5, pp. 153-181, Kluwer Academic, 1996.

11. C-K. Toh, "Associativity-Based Routing For Ad-Hoc Mobile Networks," in J. on Wireless Personal Commun., vol. 4, no. 2, 1997 , pp. 1-36.

12. A. Nandan, S. Das, G. Pau, M. Gerla, and M. Y. Sanadidi, "Cooperative downloading in vehicular ad-hoc wireless networks," in Proc. WONS'05, pp. 32-41, Jan. 2005.

13. L. Yin and G. Cao, "Supporting Co-operative Caching in Ad Hoc Networks," in Proc. IEEE INFOCOM, 2004.

14. M. Holzbock, et al., "Evolution of aeronautical communications for personal and multimedia services," IEEE Commun. Magn., vol. 41, no. 7, pp. 36-43, Jul. 2003

15. B. Karp and H. T. Kung, Greedy Perimeter Stateless Routing for Wireless Networks, in Proceedings of the Sixth Annual ACM/IEEE International Conference on Mobile Computing and Networking (MobiCom 2000), Boston, MA, pp. 243-254, August 2000.

16. Daniel Medina, Felix Hoffmann, Francesco Rossetto. " ROUTING IN THE AIRBORNE INTERNET", 2010 Integrated Communications Navigation and Surveillance (ICNS) Conference May 11-13, 2010

17. D. Medina, F. Hoffmann, S. Ayaz and C.-H. Rokitansky, Feasibility of an Aeronautical Mobile Ad Hoc Network Over the North Atlantic Corridor, IEEE SECON 2008, San Francisco, CA, June 2008.

18. SeUng Hyeon and Ki-Il Kim, Gyeongsang National University, Jinju, Republic of Korea "A NEW GEOGRAPHIC ROUTING PROTOCOL FOR AIRCRAFT AD HOC NETWORKS" 29th Digital Avionics Systems Conference October 3-7, 2010.

19. Kevin Peters, Abdul Jabbar, Egemen K. C, etinkaya, James P. G. Sterbenz "A Geographical Routing Protocol for

20. Highly-Dynamic Aeronautical Networks" 2011 IEEE Wireless Communications and Networking Conference 28-31 March 2011.

21. Wenzhe Gu, Jinglin Li, Maohui Lv, Qibo Sun, Fangchun Yang "Delay Aware Multipath Doppler Routing in Aeronautical Ad hoc Networks", 2011 14th IEEE International Conference on Computational Science and Engineering, 24-26 August.

22. D. B. Johnson, D. A. Maltz, and Y.-C. Hu. The Dynamic Source Routing Protocol for Mobile Ad Hoc Networks (DSR). Jul 2004. IETF MANET Working Group. Internet Draft.

23. Shangguang, Wang, Cunqun Fan, CaoDeng, WenzheGu, QiboSun, FangchunYang "A-GR: A novel geographical routing protocol for AANETs" Journal of Systems Architecture, Volume 59, Issue 10, Part B, November 2013, Pages 931-937

24. Dong-Woo Seo, Sok-Hyong Kim, Young-Joo Suh "System integration of GPSR and ADS-B for aeronautical ad hoc networks", Military Communications Conference, 2008. MILCOM 2008. IEEE, 16-19 Nov. 2008
25. Zhong Dong, Zhu Yian, You Tao, Duan Junhua, Kong Jie, "A new data transmission mechanism in aeronautical ad hoc network", , vol. 00, no. , pp. 255-260, 2014, doi:10.1109/BIGCOMP.2014.6741447 T. Clausen, P. Jacquet, "Optimized Link State Routing Protocol (OLSR)" in IETF: The Internet Engineering Taskforce RFC 3626 Oct 2003, Jan 2006

26. Zone Routing Protocol (ZRP), Nicklas Beijar ,2002

27. Haas, Zygmunt J., Pearlman, Marc R., Samar, P.: Intrazone Routing Protocol (IARP), June 2001, IETF Internet Draft, draft-ietf-manetiarp-01.txt

28. Haas, Zygmunt J., Pearlman, Marc R., Samar, P.: Interzone Routing Protocol (IERP), June 2001, IETF Internet Draft, draft-ietf-manetierp-01.txt

29. Haas, Zygmunt J., Pearlman, Marc R., Samar, P.: The Bordercast Resolution Protocol (BRP) for Ad Hoc Networks, June 2001, IETF Internet Draft, draft-ietf-manet-brp-01.txt

30. Haas, Zygmunt J., Pearlman, Marc R.: The Performance of Query Control Schemes for the Zone Routing Protocol, August 2001, IEEE/ACM Transactions on Networking, Vol. 9, No. 4

31. Dong Zhong, Yining Wang, Yi'an Zhu, Tao You, "An Aeronautical Ad Hoc Network Routing Protocol based on Air Vehicles Movement Features" ： 2016 22nd International Conference on Applied Electromagnetics and Communications (ICECOM), 19-21 Sept. 2016

32. Dragos Niculescu and Badri Nath "Trajectory based forwarding and its applications" 9th Annual International Conference on Mobile Computing and Networking, MobiCom '03, pages 260-272, New York, NY, USA, 2003. ACM

33. J. A. Sethian. A fast marching level set method for monotonically advancing fronts. In PROC. NAT. ACAD. SCI, pages 1591-1595, 1995

34. Urs Ramer. An iterative procedure for the polygonal approximation of plane curves. Computer Graphics and Image Processing, 1(3):244 $-256,1972$.

35. H. Labiod, N. Ababneh, and M. Garcia de la Fuente. An efficient scalable trajectory based forwarding scheme for vanets. In Advanced Information Networking and Applications (AINA), 2010 24th IEEE International Conference on, pages 600-606, April 2010

36. Qin Luo and Junfeng Wang "Multiple QoS Parameters-Based Routing for Civil Aeronautical Ad Hoc Networks " IEEE Internet Of Things Journal, Vol. 4, NO. 3, June 2017.

37. [37]. Senthil Sivakumar M, Gurumekala T, Sundaram A, Banupriya M \& Arputharaj T, 2015, 'Design of Novel AES processor for High Speed Next Generation Internet Security', International Journal of Applied Engineering Research, Vol.10 (55), pp.389-393.

38. [38]. Gurumekala T, Senthil Sivakumar M, Sundaram A \& Arputharaj T, 2015, 'Enhanced Fuzzy Based Clustering Approach for Improving Reliability of WSNs, International Journal of Applied Engineering Research, Vol.10(55), pp.1314-1319.

39. [39]. Gurumekala T, Senthil Sivakumar M, Sundaram A \& Arputharaj T, 2015, Identification of High Throughput Path in WMNs using Novel Routing Metric, International Journal of Applied Engineering Research, Vol.10 (55), pp.1278-1283.

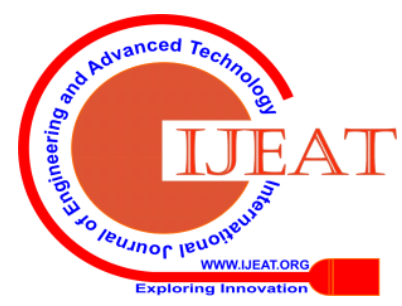

